\title{
Téoros
}

Revue de recherche en tourisme

\section{Modalités d'accès aux activités touristiques des personnes déficientes}

\section{Frédéric Reichart}

Volume 28, numéro 2, 2009

URI : https://id.erudit.org/iderudit/1024810ar

DOI : https://doi.org/10.7202/1024810ar

Aller au sommaire du numéro

Éditeur(s)

Université du Québec à Montréal

ISSN

0712-8657 (imprimé)

1923-2705 (numérique)

Découvrir la revue

Citer cet article

Reichart, F. (2009). Modalités d'accès aux activités touristiques des personnes déficientes. Téoros, 28(2), 73-81. https://doi.org/10.7202/1024810ar
Résumé de l'article

Cet article propose d'identifier les modalités d'accès aux activités touristiques pour les personnes déficientes et d'analyser les fondements idéologiques et institutionnels qui les sous-tendent. À partir d'entretiens, de textes associatifs et réglementaires, nous avons établi une bipolarisation idéologique soutenue par une solide organisation structurelle. Une logique de différenciation préconisant des loisirs touristiques sectoriels, proposés uniquement à des personnes déficientes, s'organise. Parallèlement, d'autres loisirs touristiques se développent dans une logique d'assimilation, étayés à travers un dispositif de labellisation de structures accessibles pour l'accueil de personnes déficientes. Ces deux modalités tentent de répondre à la diversité des besoins inhérents à chaque type et degré de déficience.
Ce document est protégé par la loi sur le droit d'auteur. L'utilisation des services d’Érudit (y compris la reproduction) est assujettie à sa politique d'utilisation que vous pouvez consulter en ligne.

https://apropos.erudit.org/fr/usagers/politique-dutilisation/ 


\title{
Modalités d'accès aux activités touristiques des personnes déficientes
}

\author{
Frédéric REICHHART \\ Chargé d'enseignement \\ Laboratoire en sciences sociales du sport \\ Université de Strasbourg (UDS) \\ frederic.reichhart@wanadoo.fr
}

\begin{abstract}
RÉSUMÉ: Cet article propose d'identifier les modalités d'accès aux activités touristiques pour les personnes déficientes et d'analyser les fondements idéologiques et institutionnels qui les sous-tendent. À partir d'entretiens, de textes associatifs et réglementaires, nous avons établi une bipolarisation idéologique soutenue par une solide organisation structurelle. Une logique de différenciation préconisant des loisirs touristiques sectoriels, proposés uniquement à des personnes déficientes, s'organise. Parallèlement, d'autres loisirs touristiques se développent dans une logique d'assimilation, étayés à travers un dispositif de labellisation de structures accessibles pour l'accueil de personnes déficientes. Ces deux modalités tentent de répondre à la diversité des besoins inhérents à chaque type et degré de déficience.
\end{abstract}

Mots-clés: Personnes déficientes, séjour adapté, labellisation, charte, accessibilité.

Les activités touristiques et de loisirs concernent également les personnes déficientes. De nombreuses activités, sites et infrastructures bénéficient d'aménagements et d'adaptations afin d'être accessibles à ces personnes (Reichhart, 2006). Toutefois, l'accès des personnes déficientes motrices, intellectuelles, visuelles et auditives au tourisme met en perspective une bipolarisation des propositions : un premier type de propositions résulte de prestataires spécialisés dans l'organisation d'activités touristiques pour ce public, tandis qu'un second type de propositions provient de prestataires généralistes accueillant ce public parmi leur clientèle. Sous le nom de «tourisme intégré», ce dernier se caractérise par l'aménagement d'une offre au sein du marché généraliste, impliquant une mixité entre des vacanciers déficients et des vacanciers sans déficience. L'autre modalité, intitulée «tourisme sectoriel» correspond à des séjours regroupant uniquement des vacanciers déficients. Cela alimente un questionnement autour de l'organisation de ces activités : comment s'organise et se structure chaque modalité? Il convient d'identifier et d'analyser les fondements de leur organisation institutionnelle, mais aussi idéologique.

Découlant d'un doctorat réalisé entre 2004 et 2007, cet article s'appuie sur de multiples sources; il s'agit de documents imprimés provenant d'archives internes (rapports d'activités, journaux associatifs, textes de présentation, guides, brochures d'information, dossiers de presse), d'archives administratives et légales (imprimés officiels des associations, déclarations des statuts) ainsi que d'actes de colloques, d'études de marché et d'enquêtes statistiques. Pour approfondir ces données écrites, l'auteur a complété les sources imprimées par des entretiens auprès d'acteurs dont la fonction et les actions concernant le «tourisme adapté» restent significatives (responsables de service et de secteur d'activité, présidents d'association, directeurs de structures, salariés et bénévoles).

Dans une première partie, les éléments exposés décrivent la structure institutionnelle organisant chaque modalité ainsi que les outils développés. Concernant la modalité sectorielle, il s'agit de souligner l'influence d'une mobilisation associative visant à organiser les activités. Dès les années 1980, l'absence d'un cadre réglementaire se traduit par des dysfonctionnements, entraînant de sévères critiques à propos de la qualité des séjours et qui imposent une mise en ordre. En 1991, une initiative sous la forme d'une fédéralisation des prestataires donne naissance au Conseil National des Loisirs et du Tourisme Adapté (CNLTA). Le tourisme intégré ne bénéficie pas de la même structuration que le tourisme sectoriel. Émergeant déjà dans les années 1960, il se développe différemment selon qu'il s'adresse à des enfants ou à des adultes. Dans le cadre 


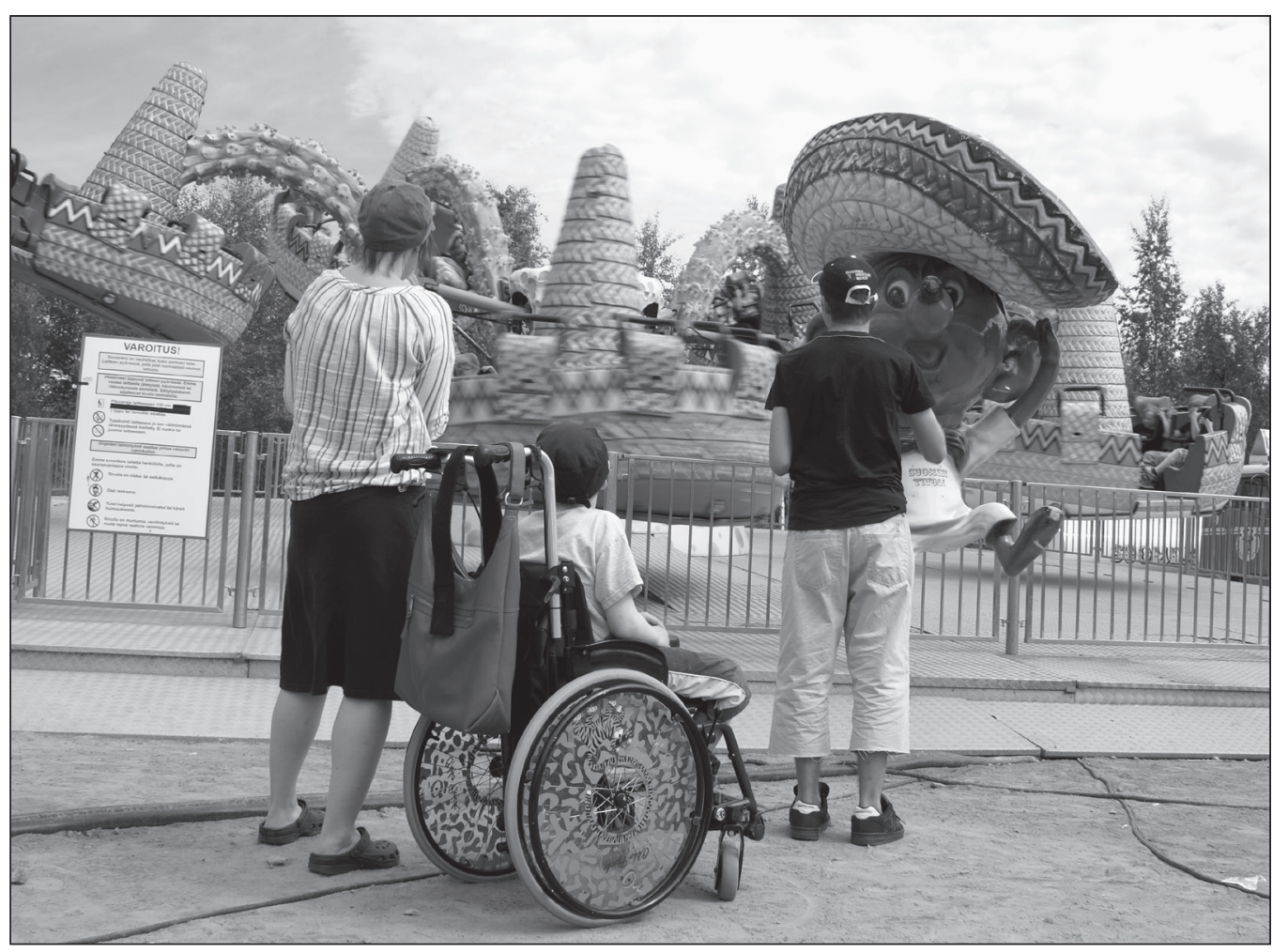

ILLUSTRATION 1 : Sans aménagements, le touriste handicapé peut être privé d'une part importante de l'expérience touristique (photo : Alain A. Grenier). des accueils mineurs, l'intégration d'enfants déficients dans les centres de vacances dits "ordinaires" reste soutenue par une association d'éducation populaire, Jeunesse au Plein Air (JPA), qui élabore dès 1997 une charte déontologique. Pour les séjours des adultes, il faudra attendre l'intervention du Secrétariat d'État au tourisme qui lance, en 1998, la première campagne annuelle de sensibilisation aux vacances des personnes handicapées. Cette politique se poursuit en 2001 par une campagne de labellisation de structures accessibles aux personnes déficientes.

Dans une seconde partie, nous allons porter une attention particulière à l'idéologie sous-jacente aux loisirs touristiques des personnes déficientes. De la même manière que l'ensemble des domaines relatifs au handicap comme le travail, la scolarité ou les pratiques sportives, l'idéologie évoquée fait référence soit à un modèle assimilatoire, soit à un modèle différentiel. Pour le premier modèle, il s'agit de mettre la déficience au second plan, de soutenir la normalisation de l'individu déficient et son inscription dans l'espace social normatif et commun. Quant au second modèle, celui de la différentiation, il soutient la spécificité de la déficience pour légitimer une prise en charge adaptée.

Enfin, la troisième et dernière partie de cet article tente de passer outre ces clivages idéologiques. Identifiant les conditions nécessaires pour garantir l'accès des personnes déficientes aux activités touristiques, elle préconise une approche écologique qui aboutit à l'ajustement des ressources de l'individu avec les exigences et contraintes de l'environnement dans lequel il évolue. De ce fait, des facteurs individuels et des facteurs environnementaux se combinent.

\section{La structuration institutionnelle des activités touristiques des personnes déficientes}

Comme le souligne l'étude de marché de l'Agence Française de l'Ingénierie Touristique (AFIT) en 2001, les loisirs touristiques des personnes déficientes se caractérisent par une bipolarité (Doucet, 2001). Parallèlement à des activités dites sectorielles uniquement proposées à des personnes déficientes, des activités dites mixtes proposent l'élargissement ou l'ouverture des activités touristiques généralistes à ce même public. Chaque modalité organisée autour d'un dispositif autonome bénéficie d'une structuration institutionnelle. Dans un premier temps, ce dispositif s'est progressivement construit à partir d'actions associatives comprenant l'élaboration d'un certain nombre d'outils tels que des chartes de qualité, des labels ou des référentiels de formation. Puis, dans un second temps, l'intervention du législateur et de l'État complète ces actions, consolidant chaque dispositif.

\section{Les loisirs touristiques intégrés}

La structuration de la modalité dite intégrée apparaît complexe, conditionnée par la combinaison de plusieurs paramètres. Soutenue par l'investissement de multiples instances associatives, mais aussi étatiques, elle se caractérise avant tout par la distinction entre un dispositif réglementant les séjours mineurs et un dispositif réglementant les séjours pour adultes. Concernant les séjours intégrés pour mineurs, correspondant à des intégrations d'enfants déficients dans des centres d'accueil pour mineurs non spécialisés, le cadre législatif en vigueur relève du ministère de la Jeunesse et des Sports, sans pour autant bénéficier d'ajustements réglementaires 
ILLUSTRATION 2 : Passerelle pour l'embarquement des personnes en fauteuil roulant dans un train à quai (photo : Frédéric Reichhart).

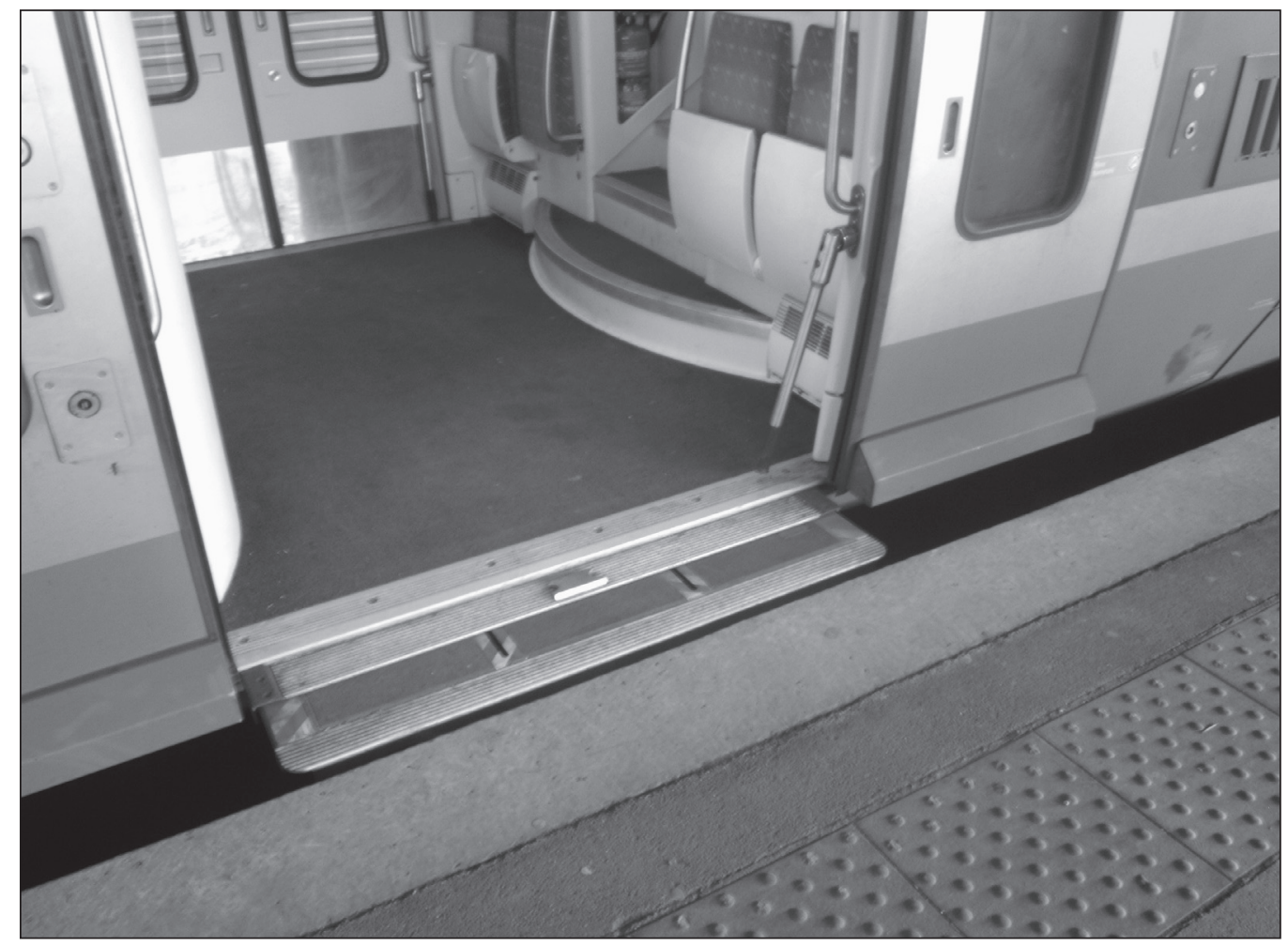

particuliers. En d'autres termes, les séjours accueillant des mineurs déficients dépendent des mêmes obligations et exigences que celles accueillant des mineurs non déficients. Dès les années 1990, des démarches et des tentatives pour structurer ces intégrations voient le jour : primo, les conditions à mettre en ouvre pour garantir la qualité des intégrations sont définies dans une charte; secundo, des formations pour l'encadrement de séjours de vacances pour mineurs sont organisées. Ces actions sont orchestrées par une association d'éducation populaire, Jeunesse au Plein Air (JPA). Dès 1992, la JPA organise une campagne de communication visant à favoriser «l'accueil en vacances de personnes handicapées en dehors du cadre institutionnel des établissements» (JPA, $2005: 4$ ). Elle soutient une autre alternative aux séjours organisés par les établissements spécialisés nommés «transferts d'établissement» ou «séjours institutionnels» ainsi qu'aux séjours sectoriels ou dits adaptés, en préconisant l'intégration d'enfants déficients dans les centres de vacances et de loisirs non spécialisés (Camberlein et al., 1982 : 125). À partir de 1993, une forte mobilisation s'engage dans cette direction avec la JPA : 15 partenaires, organisations de jeunesse, de tourisme et de loisir, comités d'entreprise et fédérations d'enseignement ou encore associations de personnes handicapées, travaillent à la rédaction d'une charte de déontologie définissant les conditions d'une intégration de qualité des personnes déficientes en vacances. La charte, lancée en juillet 1997, illustre une mobilisation des partenaires du secteur public (pouvoirs publics, collectivités territoriales ou locales, organismes sociaux), du secteur social (associations, coopératives, mutuelles) et du secteur économique au profit du développement des vacances des personnes déficientes avec des personnes sans déficience. Depuis le lancement de la charte, 19 associations en sont devenues signataires, augmentant à 45 le nombre d'organismes s'engageant à rendre effectif et concret l'intégration des personnes déficientes dans le domaine des vacances et des loisirs (JPA, 2006 : 2). Parallèlement à cette charte, des actions sont engagées dans le domaine de la formation de l'encadrement. Le Brevet d'Aptitude aux Fonctions d'Animateur de centres de vacances et de loisirs (BAFA) permet l'encadrement à titre non professionnel, de façon occasionnelle, des enfants et adolescents en centre de vacances ou de loisirs. Parmi les modules d'approfondissement de cette formation, un module s'oriente vers l'accueil des personnes handicapées.

La structuration des séjours intégrés pour adultes diffère quelque peu de celle des séjours pour mineurs, conditionnée par d'autres enjeux, notamment l'accessibilité architecturale (Sanchez, 1989). À partir des années 1990, stimulée par un canevas législatif qui impose l'accessibilité des établissements recevant du public, de la voirie et des transports, l'accessibilité apparaît autant progressivement que timidement comme une réalité concrète. L'accès à des sites touristiques tels que des musées, des restaurants, des hôtels, des parcs d'attractions pour les personnes déficientes, sans être généralisé et systématique, devient de plus en plus commun et un certain nombre de prestataires touristiques inclut les personnes déficientes dans leur clientèle ou leurs adhérents. Toutefois, ces nombreuses initiatives se caractérisent par une absence d'unité et de coordination ainsi que par une visibilité relativement réduite. Les actions réalisées se cantonnent à un niveau local, ne dépassant pas le cadre 
départemental ou régional. Par exemple, Ducher et Troubat (1999 : 19) décrivent l'élaboration d'un répertoire des structures accessibles de la Creuse : en 1999, une brochure promotionnelle éditée par le Comité Départemental du Tourisme de la Creuse répertorie l'ensemble des prestations accessibles, sous la forme de fiches techniques, validées par l'Association des Paralysés de France (APF). Le contenu de ce document sélectionne des hébergements, des établissements de restauration et des sites à visiter. Dans la même logique d'identification des sites accessibles, il nous faut également exposer une démarche dont va s'inspirer le Secrétariat d'État au tourisme en 2001, «[a]fin de rendre l'ensemble des sites touristiques et les loisirs accessibles à tous ", la région NordPas-de-Calais a lancé une opération de labellisation, sous l'égide de la délégation régionale de l'APF, du Conseil général et régional du Nord. Ce label intitulé «Le tourisme, c'est pour tous", aisément identifiable par un logo, est attribué aux structures touristiques accessibles. En 1997, 120 labels de ce type ont été attribués, près de 300 en 1998 et plus de 330 en 1999. Un autre système de pictogrammes utilisé par Village Vacances Familles (VVF) avec le partenariat de l'APF, consiste en l'application et la diffusion de sigles définissant des catégories de séjours selon leur accessibilité. D’autres labels, soutenus par des fédérations sportives, tels que le label
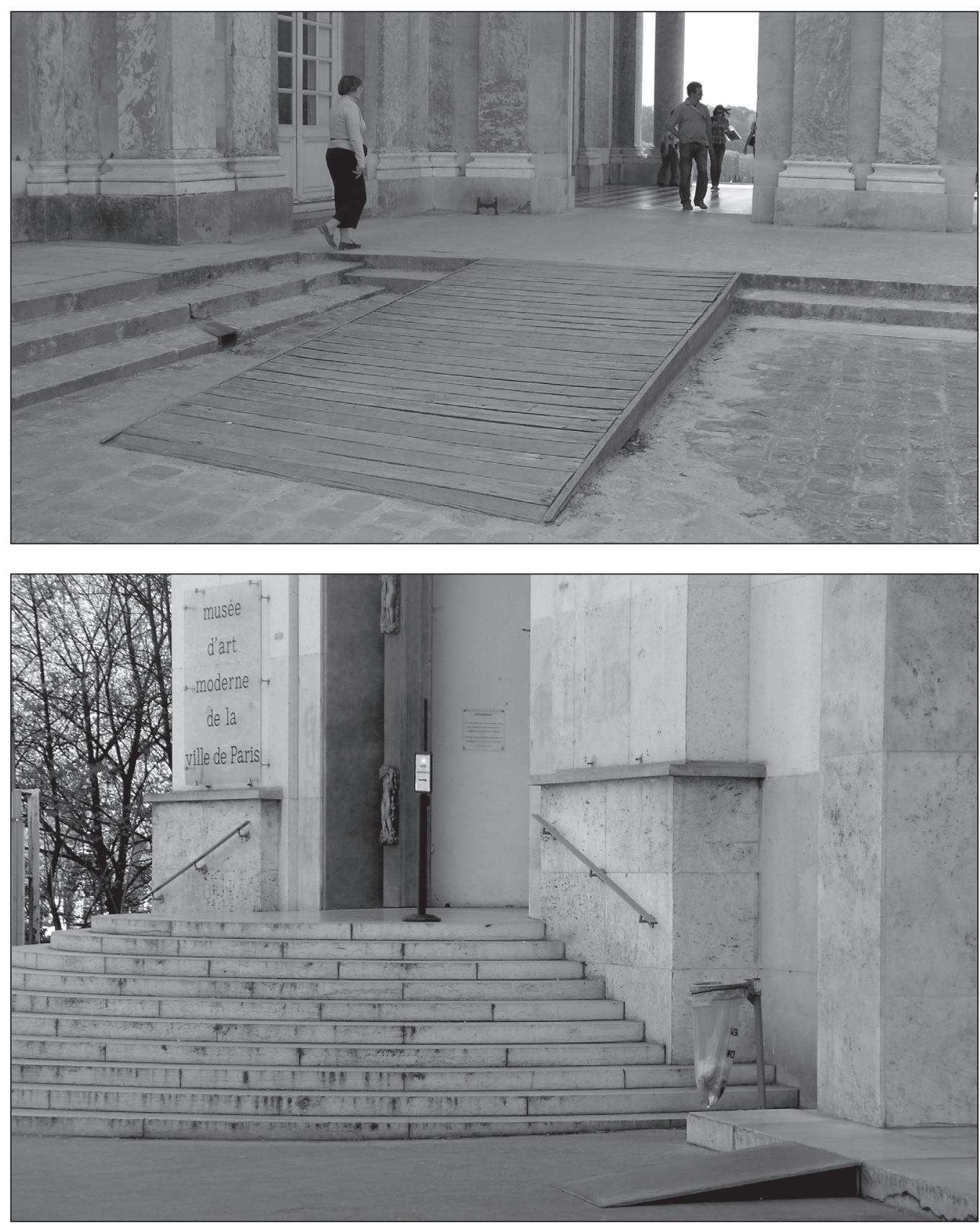

ILLUSTRATION 3 : Rampe d'accès au Château de Versailles (photo : Frédéric Reichhart).
ILLUSTRATION 4 : À côté de l'escalier d'entrée, l'accès adapté du Musée d'art moderne de la ville de Paris (photo : Frédéric Reichhart). 
Espace Loisir Handisport (ELH), le label Handivoile, le label Activités Équestres Adaptées ou encore le label Hand Icare se côtoient. Depuis quelques années, un label Handiplage destiné à identifier les plages accessibles existe également (Handiguide, 2006 : 163). Tous ces labels témoignent d'une mise en accessibilité de sites et d'activités à des personnes déficientes. Pourtant, aucun label ne revêt une dimension véritablement nationale et aucun répertoire ne recense l'ensemble des structures au niveau de l'ensemble de l'hexagone. Les labels se sont développés sans parvenir à se coordonner, n'aboutissant pas à un label fédérateur; à l'instar, les guides, de plus en plus nombreux, se multiplient.

À partir de 1998, sous l'impulsion du Secrétariat d'État au tourisme et les recommandations d'un rapport d'expertise (Gagneux, 1999), une politique globale voit le jour. C'est dans ce cadre qu'une campagne nationale de labellisation débute en 2001 : elle consiste à identifier un organisme de vacances ou une structure accessible aux personnes déficientes par l'obtention d'un label. Ce label, certifiant la qualité de l'accueil proposée à sa clientèle, demeure contractualisé par la signature d'une charte : le premier label fut attribué à la cité des sciences et de l'industrie le 4 mai 2001 (Vivre ensemble, 2003). Cette campagne de labellisation est confiée à une association regroupant des institutions et des représentants du milieu touristique, mais aussi du secteur du handicap, nommée Association Tourisme et Handicaps (ATH). La mise en place de ce label traduit le choix d'une stratégie volontariste. Si les politiques antérieures de promotion de l'accessibilité des sites s'appuyaient sur l'obligation affirmée par un canevas législatif, la demande d'attribution du label ATH constitue une démarche volontaire : elle découle nécessairement du souhait et de la motivation des prestataires de service et des professionnels à s'engager de façon durable et qualitative dans l'accueil de clients déficients. Attribué pour un, deux, trois ou quatre types de déficience selon les conditions d'accessibilité présentes, le label souligne que l'accessibilité ne demeure pas homogène et uniforme et qu'elle varie selon les déficiences. Les adaptations et les aménagements nécessaires, qu'ils soient liés à l'environnement humain, en termes de sensibilisation et de formation du personnel, ou à l'environnement architectural, en termes d'accès et de signalétique, diffèrent. D'après les données de l'Association Tourisme et Handicaps, les structures labellisées ATH accessibles aux personnes déficientes mentales sont majoritaires, proches de $79 \%$ du total des équipements labellisés. Elles sont suivies de près par celles accessibles aux personnes déficientes motrices, qui composent $65 \%$ des labels. Quant aux structures accessibles aux déficiences sensorielles, elles restent les moins nombreuses : elles sont de $43 \%$ pour les déficiences auditives et de $25 \%$ pour les déficiences visuelles. D'un point de vue numéraire, en août 2007, seulement 2385 équipements et sites sont labellisés ATH (Tourisme et handicaps, $2007: 6$ ). D'après le rapport de la direction du Tourisme (direction du Tourisme, $2006: 41$ ) présenté à la commission des comptes du tourisme en 2005, 231000 entreprises composent les activités d'hôtellerie, de restauration et de loisirs du secteur touristique français. L'aménagement des sites et équipements dans le but de favoriser l'accès aux personnes déficientes ne concerne qu'une infime partie du parc touristique.

\section{Les loisirs touristiques sectoriels}

Quant au tourisme sectoriel, il apparaît et se développe dans un autre contexte. Dans les années 1980-1990, des associations diverses et variées telles que ASLV-Tourisme Adapté, Euro Évasion Vacances adaptées, Nouvelles Évasions Voyages adaptés voient le jour, composant l'offre des séjours dit adaptés. Il s'agit de séjours organisés exclusivement pour des personnes déficientes. Avant la loi no 92-645 du 13 juillet 1992 fixant les conditions d'exercice des activités relatives à l'organisation et à la vente de voyages ou de séjours dite «loi tourisme» et la loi $\mathrm{n}^{\circ}$ 2005-102 du 11 février 2005 pour l'égalité des droits et des chances, la participation et la citoyenneté, ces séjours n'étaient assujettis à aucun texte législatif. L'article 48 impose la possession d'un agrément "vacances adaptées organisées», délivré par les autorités préfectorales. Dans ce vide réglementaire, la croissance et la multiplication de ces séjours sectoriels s'accompagnent de dérives et de dysfonctionnements importants qui ternissent l'image du «tourisme adapté». Tant au niveau qualitatif et quantitatif de l'encadrement que des conditions matérielles mobilisées pendant le séjour, de nombreux efforts restent à faire pour redorer son blason. C'est dans cette obligation et contrainte que ces activités doivent se structurer, s'organiser. En 1990, la mobilisation de certains organisateurs et prestataires de séjours aboutit à la création d'une association, le Conseil National des Loisirs et du Tourisme Adapté (CNLTA) : la mission de cette instance consiste à fédérer la multitude d'opérateurs intervenant dans ce domaine, mais aussi à organiser des prestations de qualité. Par contre, ne parvenant pas à rassembler tous les prestataires, le CNLTA n'apparaît pas représentatif de l'ensemble du «tourisme adapté».

Au fil des années, le CNLTA va occuper une place de plus en plus prépondérante dans la modalité que nous nommons tourisme sectoriel. Il va particulièrement organiser et fédérer les activités touristiques en faveur des personnes déficientes mentales. Le CNLTA se constitue comme une instance fédérative avec une mission de rassemblement, mais aussi comme une instance paritaire : les structures adhérentes au CNLTA, au nombre de 42 , se composent d'associations représentantes d'usagers (25) et des organisateurs prestataires de vacances (17). Les critiques concernant les activités de tourisme sectoriel, relatives à la qualité des séjours, poussent le CNLTA à se mobiliser afin de rehausser le niveau qualitatif des prestations. Dans cette perspective, il entame une politique de changement et entreprend une démarche de qualité à travers plusieurs dispositions. Tout d'abord, il met en place une grille d'évaluation pour constituer des groupes homogènes en fonction de l'autonomie des vacanciers afin de définir un taux d'encadrement. Ensuite, il élabore une charte de qualité et un dispositif de contrôle d'application de celle-ci. Enfin, il établit un module de formation destiné à l'encadrement des séjours.

Un premier outil visant à structurer et organiser les séjours renvoie à la grille d'évaluation que propose le CNLTA à ses adhérents. Cette grille s'appuie sur l'identification du profil des participants à partir de leur potentiel pour composer des groupes homogènes (CNLTA, 2004 : 5). Il s'agit d'établir le profil de la personne en identifiant ses capacités physiques et mentales afin de définir un niveau d'autonomie qui détermine un nombre pré-établi et suffisant de personnel. En complément 
de la régulation du taux d'encadrement suivant l'autonomie du vacancier, le CNLTA développe d'autres mesures répondant à l'objectif de garantir un niveau qualitatif, comme la charte nationale de qualité du CNLTA. Officiellement lancée le 22 septembre 1999 au Palais des Congrès à Paris, elle annonce un changement majeur : «Professionnaliser le tourisme pour adultes handicapés mentaux et faire barrage à l'amateurisme ou aux marchands du temple qui menacent le secteur, tels sont les principaux objectifs de la charte de qualité élaborée sous l'égide du CNLTA.» (ASH, 1998 : 22) Incarnant qualité et rigueur, elle tente d'éliminer les dérives imputées à une image d'amateurisme du fait de structures peu sérieuses. En fait, la charte revêt une mission de protection et de garantie visant "la sécurité et un accueil de qualité à un public fragilisé parfois victime d'organismes peu scrupuleux» (ASH, 2000:8). Acte fort et symbolique visant à distinguer les professionnels des amateurs, elle propose d'opérer une sélection parmi les nombreux opérateurs du champ du tourisme sectoriel en identifiant ceux qui se placent du côté des professionnels et de la qualité.

La situation vis-à-vis de l'encadrement a été l'un des éléments conduisant à la création du CNLTA en 1990. Rappelons que dès l'apparition des premiers séjours pour personnes déficientes, les séjours ont été fortement critiqués par rapport à la qualité et quantité de l'encadrement. Le CNLTA envisage une solution à ce problème en 1999 avec la mise en place de la charte nationale qui comporte notamment les fondements d'une politique de formation. Dans cette logique, un référentiel de formation est élaboré à partir de 2002 (CNLTA, $2002: 2$ ). Il rappelle que la charte impose des conditions concernant l'encadrement, mais aussi la formation du personnel encadrant. Le CNLTA alimente un débat autour du statut, de la fonction, de la formation et de la rémunération de l'encadrement. Il soutient la création d'un statut d'animateur-accompagnateur, qui bénéficie d'une formation, mais ne pouvant prétendre à une rémunération, car cette dernière fragilise l'équilibre financier des séjours. Ce débat sera tranché et prendra fin avec l'article 17 de la loi du 23 mai 2006 relative au volontariat associatif et à l'engagement éducatif. L'encadrement des séjours pour personnes déficientes s'inscrit sous un statut et des conditions (rémunération minimum, temps et durée de travail, etc.) précisées dans le cadre de l'engagement éducatif.

\section{Les fondements idéologiques : entre assimilation et différenciation}

Les activités touristiques des personnes déficientes convoquent et révèlent des idéologies divergentes : entre les activités touristiques sectorielles et les activités touristiques intégrées, deux conceptions de l'altérité, de la cohésion sociale et du vivre-ensemble se dévoilent. Comme l'affirme l'anthropologue Henri-Jacques Stiker (1997 : 189), «la question de la déficience $[\ldots]$ constitue un test important, un point critique pour réfléchir à la capacité d'intégration [de nos démocraties] et à ses manques [...] Plus encore, elle interroge notre rapport à l'autre, notre rapport à la vie, les fondements de notre vivre-ensemble». En fait, la thématique du handicap questionne l'importance de l'altérité dans nos sociétés : il s'agit de construire une société, en tant que mode d'organisation et de relations, composée d'individus tous différents, tout en garantissant le vivre-ensemble. À leur manière, Gardou et Poizat (2007 : 211) synthétisent les enjeux et les difficultés soulignés par Stiker (1997) qui convoque les concepts de singulier et d'universel en les formalisant sous cette question : comment articuler le singulier que constitue le handicap avec l'universel de l'Homme? La réponse à cette question se concrétise par deux postures idéologiques décrites par Stiker dans la revue Esprit : «[D]eux grandes tendances se font jour dans la question de la relation à l'autre : le modèle de la différenciation et celui de l'assimilation.» (Stiker, 1999 : 195) À l'instar des pratiques sportives, des politiques de l'emploi, de la scolarisation et des modalités d'hébergement, les activités touristiques et de loisirs se concrétisent autour de finalités soient assimilatrices, soient différenciatrices. Deux approches opérationnelles et idéologiques se distinguent et s'organisent.

La différenciation, préconisant la reconnaissance des singularités de chacun, prône les différences entre les êtres humains. Dans le domaine du handicap, l'approche différentielle insiste sur la prise en considération des conséquences fonctionnelles qui proviennent des altérations organiques; elle aboutit à la nécessité de dégager des moyens spécifiques pour répondre aux besoins et aux difficultés résultant des déficiences. Selon Stiker (1999 : 189), la différenciation à l'extrême comporte des effets pervers et négatifs. En insistant trop sur la déficience, cela peut conduire à la stigmatisation et aboutir à l'exclusion des individus : la déficience devient le critère dominant, parfois unique sur lequel se fondent le statut et le rôle de l'individu (Goffman, 1975). Dans ce cas, la personne déficiente se trouve alors réduite à sa déficience. L'autre conception de l'altérité, celle de l'assimilation, issue du postulat universaliste, renvoie à un modèle normalisateur. Autant le modèle différentialiste défend les singularités et les différences individuelles, autant le modèle assimilatoire soutient l'idée de l'universalité de l'humanité et de l'unité du genre humain. Comme l'affirme Stiker (2003: 23), dans l'assimilation «l'autre ne peut être qu'un autre moi-même». Il y a risque de confusion entre les individus avec un déni des singularités. La figure de l'être humain devient homogène, uniforme. L'assimilation à l'excès peut aboutir à la recherche de l'identique et conduire au déni de l'individualité de l'autre. Dans ce cas, l'autre devient semblable et se destine à devenir moi : l'alter fusionne et se confond avec l'ego. Ce modèle idéologique risque alors d'alimenter la dictature de l'uniformité et du conformisme : l'individu, dépossédé de ses caractéristiques personnelles, perd son identité singulière et se conforme pour se fondre dans un ensemble homogène et normatif.

En confrontant le modèle idéologique de l'assimilation avec les personnes déficientes, de nombreuses questions se posent. Si nous reprenons le schéma établit par Wood en 1975, qui se décline selon la trilogie déficience-incapacité-désavantage social (ou handicap), nous définissons la déficience à un niveau strictement organique comme de «toute perte de substance ou altération d'une fonction ou d'une structuration psychologique, physiologique ou anatomique» (Wood, 1988). Quant à l'incapacité, elle se situe au niveau de l'individu, en termes d'actions. Définie par Wood comme «toute réduction [résultant d'une déficience] partielle ou totale de 
la capacité d'accomplir une activité d'une façon, ou dans des limites considérées comme normales pour un être humain ", elle correspond aux gênes ou impossibilités rencontrées par la personne pour réaliser des actes de la vie quotidienne (Wood, 1988). En somme, du fait de leurs altérations organiques et fonctionnelles, les personnes déficientes présentent des singularités très marquées, qui se répercutent sur la vie quotidienne, en termes d'incapacités et de difficultés. Par exemple, une personne amputée de ses deux jambes (altération organique) éprouvera des difficultés motrices en termes de déplacement (incapacité) qui peuvent se répercuter au niveau des activités sociales et professionnelles de la personne (désavantage social). Au nom de l'universalisme, peut-on nier ces singularités et ces difficultés? Peut-on considérer une personne handicapée comme identique et semblable à une personne intègre, au-delà de la singularité inhérente aux conséquences de sa déficience? Ne faut-il pas prendre en compte cette spécificité? Cela suppose de considérer les personnes déficientes comme des personnes ayant besoin d'être reconnues et traitées en fonction de leurs altérations organiques, par conséquent comme des individus uniques et différents. Par-delà les clivages conceptuels et théoriques, il convient de parvenir à concilier la différenciation et l'assimilation, dans des modalités opérationnelles et appliquées. La finalité visée consiste simplement à garantir aux personnes déficientes la possibilité d'accéder aux activités touristiques.

\section{Quelles conditions pour l'accès au tourisme?}

Afin de concrétiser cette finalité dans le cadre des activités touristiques intégrées ou sectorielles, des conditions s'avèrent indispensables. Pour aborder ces conditions, les modèles écologiques privilégiant l'articulation des individus et de l'environnement semblent intéressants. De manière plus précise, l'approche conceptualisée par l'anthropologue canadien Patrick Fougeyrollas dans le Processus de production du handicap (PPH), en soutenant l'interaction de facteurs individuels et de facteurs environnementaux, peut s'avérer utile pour préciser ces conditions (1998). En effet, la convocation de cette approche légitime la distinction de conditions internes ou intrinsèques et de conditions externes ou extrinsèques garantissant l'accès des personnes déficientes aux loisirs touristiques.

\section{Des conditions internes}

Lorsque nous parlons de conditions internes, nous pouvons faire référence aux facteurs personnels présentés dans le schéma du PPH. Ces facteurs renvoient aux caractéristiques appartenant à la personne telles que l'âge, le genre, l'identité culturelle, etc., et se déclinent sous une dimension organique et fonctionnelle : le système organique comprenant l'ensemble de composantes corporelles, qui oscille de l'intégrité physique à la déficience, demeure complété par les aptitudes regroupant les capacités et incapacités de l'individu. En somme, les facteurs personnels se composent du potentiel organique, mais aussi fonctionnel de la personne. Nous retrouvons dans ces facteurs l'affirmation d'une vision essentiellement biologique et motrice du corps affirmée notamment dans le modèle médical de Wood (1988). Pour les personnes déficientes, ce potentiel peut être compensé et reste proportionnel à un niveau d'autonomie : plus le potentiel de la personne est important, plus le degré d'autonomie est élevé. Par contre, nous ajoutons à ce potentiel une dimension psychologique notamment motivationnelle. La motivation de l'individu constitue une condition importante pour l'accès au tourisme qui ne demeure pas constante ou omniprésente. Certaines personnes déficientes ou intègres n'émettent pas le souhait de bénéficier de pratiques touristiques. Pour les personnes déficientes, la question de la motivation apparaît déterminante, car elle implique une inscription dans un type de séjour : si certaines personnes déficientes préfèrent bénéficier des séjours sectoriels, d'autres affichent une préférence pour les séjours intégrés. Cette affirmation contredit l'idée réductrice d'une demande systématique des personnes déficientes d'accéder à des loisirs touristiques sectoriels. Toutefois, la motivation, bien qu'essentielle et déterminante, reste une condition limitée, dans le sens où elle ne suffit pas à garantir l'accès. Vouloir partir en séjour intégré ne suffit pas, il faut également «le pouvoir» en termes en d'aptitudes : la dimension psychologique rejoint la dimension motrice et corporelle. Afin d'illustrer cet aspect, considérons deux illustrations. La première illustration s'appuie sur les séjours sportifs de type «treks» ou randonnées dans le désert ou en montagne. L'inscription à ce type de séjours demeure corrélée à une exigence de niveau, concrétisée par la possession et la maîtrise d'aptitudes de chaque participant. Il ne s'agit pas de constituer un groupe parfaitement homogène, mais plutôt de s'assurer que le participant possède le potentiel suffisant pour répondre aux exigences inhérentes au séjour et par conséquent à y participer. Pour se faire, un système d'identification des difficultés établi à partir du dénivelé, du portage, du confort de l'hébergement, de l'expérience des participants, de la durée de marche quotidienne, des capacités techniques (piolet, cramponnage, encordage) et physiques vise à coordonner le niveau des «trekkeurs» avec les exigences du séjour. Les vacanciers ne possédant pas le niveau demandé ne peuvent pas s'inscrire à ce séjour et doivent s'orienter vers un séjour adapté à leur niveau, c'est-à-dire dont les exigences minimales correspondent à leur potentiel. La seconde illustration s'inspire de la première en se transposant à des personnes déficientes. Prenons le cas, par exemple, d'une personne déficiente souhaitant s'inscrire à un séjour intégré. Cette inscription reste possible sous la condition que son potentiel, en termes de capacités, correspond aux exigences du séjour. Dans le cas contraire, si le potentiel ne correspond pas, elle ne peut pas s'y inscrire. Certaines personnes déficientes possèdent des capacités et un niveau d'autonomie suffisant pour participer à un séjour intégré, mais elles ne le souhaitent pas. La situation inverse peut également se produire : un vacancier peu autonome, possédant des capacités limitées peut avoir la motivation de s'inscrire dans un séjour intégré. Aptitudes et motivation se distinguent. Ces deux exemples traduisent la nécessité pour le vacancier non seulement d'être motivé par le type de séjour, mais également de posséder, de maîtriser, les compétences indispensables et exigées pour participer aux activités afin de dépasser les contraintes du séjour. Nous voyons qu'un autre type de conditions, des conditions "extérieures » au vacancier, émerge pour concrétiser l'accès au tourisme. 


\section{Aux conditions externes....}

En effet, l'accès au tourisme ne demeure pas uniquement conditionné par des facteurs personnels; ces exemples mettent en perspective l'influence de conditions que nous nommons «externes» et qui renvoient aux facteurs «environnementaux », en tant que « dimension sociale ou physique qui détermine l'organisation et le contexte d'une société» (Fougeyrollas, 2001). En fait, les facteurs environnementaux englobent les dimensions extérieures à l'individu. Toutefois, ils ne se restreignent pas simplement à l'environnement physique et matériel, cantonnés à la question de l'accessibilité architecturale. Ils prennent également en compte l'environnement humain, c'est-à-dire les attitudes, les représentations et la présence des individus. "Cela englobe les «barrières culturelles» en tant qu'obstacles à l'intégration et à la pleine participation des personnes handicapées dans tous les aspects de la vie sociale» (Fardeau, 2001), mais aussi l'intervention humaine. Dans notre cas, l'environnement physique implique l'accessibilité architecturale des sites et des équipements, c'est-à-dire l'aménagement de rampes, l'installation d'ascenseurs, la mise en place de signalétique, mais aussi l'utilisation de matériels adaptés tels que des fauteuils de baignade et de randonnée, par exemple. Quant à l'environnement humain, il intervient dans l'accueil et l'encadrement des touristes déficients. Il nécessite une sensibilisation du personnel d'accueil et d'encadrement aux questions relatives aux handicaps, mais aussi une formation orientée vers l'acquisition de savoir-faire techniques et comportementaux (Reichhart, 2008).
Ainsi, outre la motivation et les aptitudes des vacanciers, d'autres facteurs résultant de la structuration et de l'organisation de l'environnement physique et humain sont à prendre en compte pour l'accès au tourisme des personnes déficientes. De ce fait, il convient d'ajuster le potentiel et les ressources de l'individu avec les contraintes et les exigences de l'environnement.

Comme le souligne l'illustration 5 , la combinaison de facteurs internes regroupant les compétences et la motivation des vacanciers avec les facteurs externes impliquant l'environnement apparaît déterminante pour accéder à un séjour touristique. Par exemple, une faible autonomie du vacancier conjuguée avec des facteurs environnementaux peu «facilitateurs», c'est-à-dire un environnement architectural peu ou pas accessible, ou l'absence d'accompagnement humain particulier, constituent des obstacles pour accéder aux activités touristiques. Toutefois, ce même vacancier peut bénéficier de séjours touristiques à condition que les facteurs externes demeurent facilitateurs : cela nécessite des conditions compensant la faible autonomie du vacancier telles qu'un site architecturalement accessible, la présence d'un personnel formé ou encore du matériel adapté. Il faut se préserver d'opposer les modalités sectorielles et intégrées pour plutôt les concevoir dans une complémentarité répondant aux différences de potentialité d'accueil de l'environnement et à la variabilité des déficiences. Les loisirs touristiques intégrés et les loisirs touristiques sectoriels restent à considérer dans une continuité complémentaire au dépend d'une rupture et d'une opposition entre deux modalités. Il s'agit plutôt d'un continuum ou d'une dialectique que de deux blocs séparés.

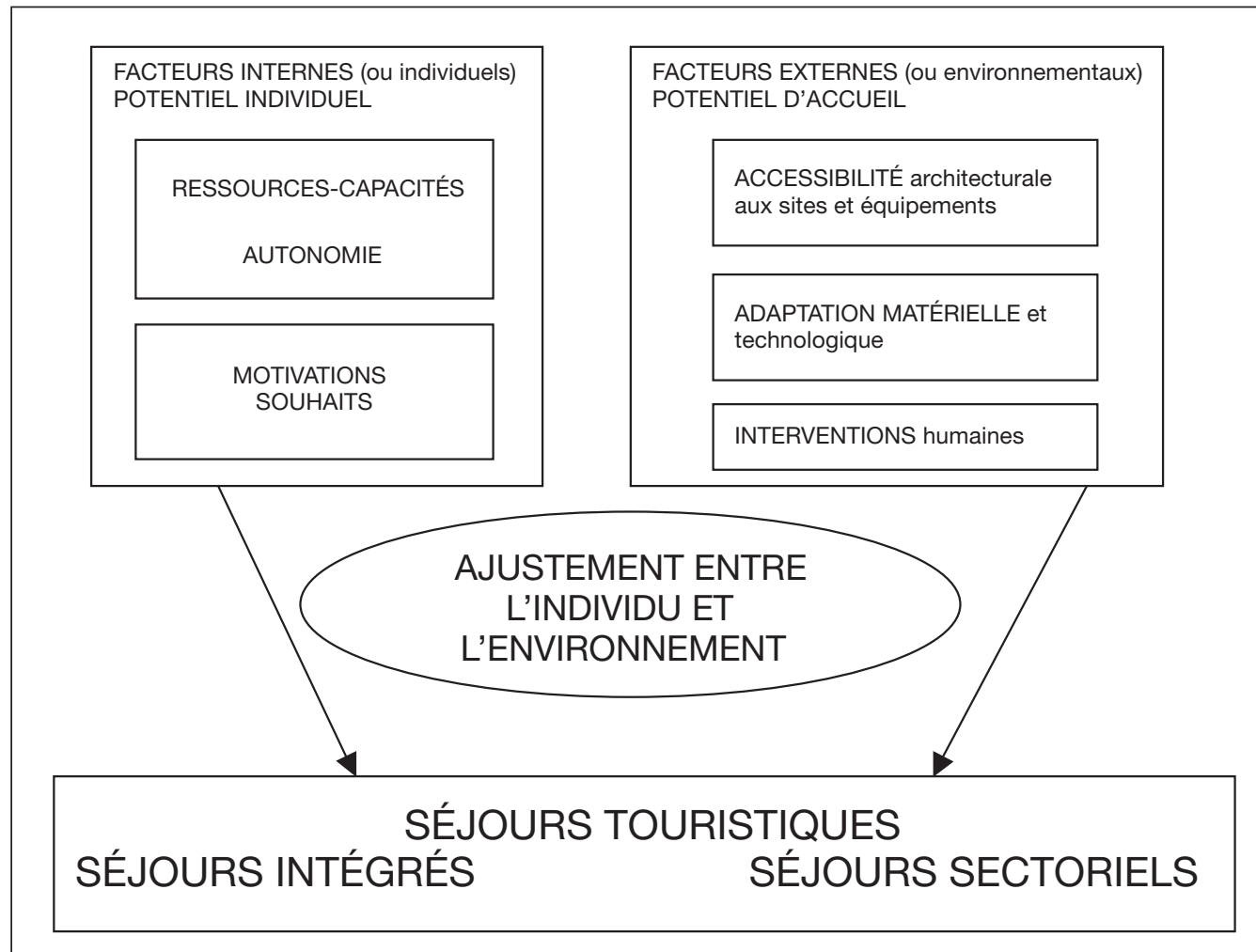

ILLUSTRATION 5 : Ajustement individu-environnement (source : Données compilées par l'auteur). 


\section{Conclusion}

Cet article, qui met en perspective l'hétérogénéité à la fois institutionnel et idéologique du champ des loisirs touristiques des personnes déficientes, insiste également sur la combinaison des conditions requises pour garantir cet accès. Les difficultés rencontrées par une personne déficiente mentale ne demeurent pas semblables à celles rencontrées par une personne déficiente motrice. Certaines déficiences nécessitent des aménagements de sites et d'activités tels que la mise en place de signalétique, la mise à disposition de matériel adapté, l'accessibilité architecturale de l'espace. D'autres déficiences imposent une intervention humaine supplémentaire. Par exemple, pour les personnes déficientes motrices, les conditions apparaissent multiples : elles convoquent un minimum d'accessibilité à l'environnement physique, mais aussi un encadrement, particulièrement pour les personnes polyhandicapées. Pour ces dernières, il nous faut noter la prépondérance du matériel de type fauteuil électrique qui permet à la personne d'être le plus autonome possible. L'innovation technologique en termes de matériel ouvre de nouveaux espaces de pratiques, notamment des activités de pleine nature comme la randonnée (avec l'invention du fauteuil tout terrain) et le ski (avec l'invention du ski-fauteuil). Ces conditions varient non seulement en fonction du type de déficience, mais également selon le degré de déficience.

Ensuite, le tourisme des personnes déficientes n'apparaît pas comme un champ autonome, mais plutôt comme un espace pluriel et polymorphe traversé de logiques divergentes : ces logiques peuvent être économiques, se rapprochant d'une logique de marché, mais elles peuvent être également politiques et sociales, étayées par des enjeux comme l'intégration, l'accessibilité, mais aussi l'encadrement et la formation du personnel. Cette diversité des logiques et des enjeux souligne la forme hétérogène de ce tourisme, représenté à tort comme uniforme. À ce titre, le pluriel paraît plus approprié que le singulier pour définir ces activités. Il semble plus précis de parler des activités touristiques des personnes déficientes que de tourisme adapté.

Pour finir, les loisirs des personnes déficientes traduisent les limites du vivre-ensemble ainsi que l'idéalisation de la cohésion sociale d'une société égalitaire. Mais ils dévoilent surtout la peur de la différence qui détermine des conduites de rejet individuelles et institutionnelles. En ce sens, ils témoignent de la difficulté de penser et de construire un monde pour tous, de s'éloigner de la norme, de la moyenne et d'inclure les écarts types dans nos pratiques et nos représentations.

\section{Bibliographie}

Actualités Sociales Hebdomadaires (2000) «Vacances et loisirs pour personnes handicapées ", Cahier II, n 2160,31 mars.

Actualités Sociales Hebdomadaires (1998) «Une charte de qualité pour le tourisme adapté», $\mathrm{n}^{\circ} 2070,8$ mai.

Association des Paralysés de France, Le tourisme, c'est pour tous, Service accessibilité, 211, rue nationale, 59800 Lille.

Association Tourisme et Handicaps (2007) Bulletin de liaison, $\mathrm{n}^{\circ} 17$ (supplément), mars.

CAMBERLEIN, Dominique; Philippe CAMBERLEIN; Jacques CRINON et Paul DURNING (1982) Enfants handicapés en centre de vacances: vers l'intégration, Paris, Édition du scarabée. 152 p.
CNLTA (2004) Dossier visiteur, Mission-Évaluation-Qualité.

CNLTA (2002) Tronc commun de formation de l'animateur-accompagnateur de séjour de vacances adaptées, Programme expérimental approuvé par le conseil d'Administration du CNLTA le 28 février.

Direction du Tourisme (2006) Les comptes du tourisme. Compte 2005. Rapport présenté à la Commission des Comptes du Tourisme, mai.

DOUCET, Alain (2001) Tourisme et handicaps : Étude de marché de la population handicapée face à l'offre touristique Française, Collection Guide de savoir-faire, Éditions AFIT. 104 p.

DUCHER, Isabelle et Jean-Pierre TROUBAT (1999) «La creuse se bat pour la liberté d'accéder partout ", Revue Espace, n 161, p. 18-21.

FARDEAU, Michel (2001) Personnes handicapées: Analyse comparative et prospective du système de prise en charge, Rapport au Ministre de l'Emploi et de la Solidarité et au Secrétaire d'État à la Santé, à l'Action Sociale et aux Handicaps, sur une analyse comparative et prospective du système français de prise en charge des personnes handicapées, Direction Générale des Affaires Sociales (DGAS).

FOUGEYROLLAS, Patrick (2001) «Le processus de production du handicap : l'expérience québécoise", dans Raphaël de Riedmatten, Une nouvelle approche de la différence. Comment penser la différence?, Genève, Édition médecine et Hygiène, p. 101-122.

FOUGEYROLLAS, Patrick; René CLOUTIER; Hélène BERGERON; Jacques CÔTÉ et Ginette ST-MICHEL (1998) Classification québécoise. Processus de production du handicap. Québec, RIPPH/SCCIDIH.

GAGNEUX, Michel (1999) Tourisme et handicap, l'offre touristique. Secrétariat d'État au tourisme, Conseil National du Tourisme. 48 p.

GARDOU, Charles et Denis POIZAT (2007) Désinsulariser le handicap, Quelles ruptures pour quelles mutations culturelles? Lyon, Connaissances de la diversité. 360 p.

GOFFMAN, Erving (1975) Stigmate. Les usages sociaux des handicaps, Paris, Le Sens commun, Éditions de Minuit. 175 p.

Handiguide Finistère (2006) Les activités physiques et sportives, ministère de la Jeunesse, des Sports et de la Vie associative.

Jeunesse au Plein Air (2006) Dossier de presse, novembre.

Jeunesse au Plein Air (2005) Vacances, loisirs collectifs et handicap : question de laïcité. Actes des XI rencontres nationales de la Jeunesse au Plein Air, 19 janvier, Paris.

REICHHART, Frédéric (2008) $10^{e}$ Congrès de l'Association Internationale de Recherche sur le Handicap Mental. Actes réunis par Viviane Guerdan: Participation des personnes en situations de handicap et tourisme.

REICHHART, Frédéric (2006) «L'offre touristique en faveur des personnes en situation de handicap : entre le tourisme intégré et le tourisme sectoriel », Traverse Revue d'histoire, novembre, p. 125-136.

SANCHEZ, Jésus (1989) Accessibilité : support concret et symbolique de l'intégration. Paris, Centre Technique National d'Études sur les Handicaps et les Inadaptations. $175 \mathrm{p}$.

STIKER, Henri-Jacques (2003) «Sens de la construction du régime de handicap vers son dépassement» dans «La compréhension sociale du handicap ", Cahier de recherche, ${ }^{\circ} 182$, Centre de Recherche pour l'Étude et l'Observation des Conditions de Vie (CREDOC), p. 15-29.

STIKER, Henri-Jacques (1999) «Quand les personnes handicapées bousculent les politiques », Revue Esprit, n 12, décembre, p. 75-105.

STIKER, Henri-Jacques (1997) Corps infirmes et sociétés, Paris, Dunod. 217 p. Vivre ensemble (2003) Faciliter les loisirs, n 65, août.

WOOD, Philip (1988) Classification internationale des handicaps : déficiences, incapacités et désavantages. Un manuel de classification des conséquences des maladies, Paris : INSERM/CTNERHI, PUF, 1988. 350 p. 